\title{
Resultados a curto prazo de ceratotomia lamelar pediculada (LASIK) para correção de hipermetropia com o sistema Ladar Vision de excimer laser
}

\author{
Short-term results of hyperopic laser in situ keratomileusis(LASIK) with the Ladar \\ Vision excimer laser system
}

\author{
Larissa Madeira Nunes ${ }^{1}$ \\ Cláudia Maria Francesconi ${ }^{2}$ \\ Mauro Campos ${ }^{3}$ \\ Paulo Schor ${ }^{4}$
}

Trabalho realizado no Setor de Cirurgia Refrativa do Departamento de Oftalmologia da Universidade Federal de São Paulo - UNIFESP.

' Pós-graduanda nível Doutorado e Médica colaboradora do Setor de Cirurgia Refrativa do Departamento de Oftalmologia da Universidade Federal de São Paulo UNIFESP.

${ }^{2}$ Médica colaboradora do Setor de Cirurgia Refrativa do Departamento de Oftalmologia e Doutora pela Universidade Federal de São Paulo - UNIFESP.

Doutor em Oftalmologia e Chefe do Setor de Cirurgia Refrativa do Departamento de Oftalmologia da Universidade Federal de São Paulo - UNIFESP.

${ }_{5}^{5}$ Doutor em Oftalmologia e Chefe do Setor de Bioengenharia Ocular do Departamento de Oftalmologia da Universidade Federal de São Paulo - UNIFESP.

Endereco para correspondência: Larissa Madeira Nunes, R. Leôncio de Carvalho 166, apt.124 - São Paulo (SP) CEP 04003-010

E-mail: lmbnunes@terra.com.br

Recebido para publicação em 14.01.2003

Versão revisada recebida em 22.05.2003

Aprovação em 27.06.2003

\begin{tabular}{|c|}
\hline RESUMO \\
\hline $\begin{array}{l}\text { Objetivo: Avaliar a eficácia e a segurança do LASIK (ceratotomia lamelar } \\
\text { pediculada) hipermetrópico utilizando-se o sistema Ladar Vision de excimer } \\
\text { laser. Métodos: Foram analisados, retrospectivamente, } 28 \text { olhos de } 17 \\
\text { pacientes com hipermetropia de }+1,00 \mathrm{a}+3,00 \mathrm{D}(\mathrm{Grupo} 1) \text { e } 29 \text { olhos de } 18 \\
\text { pacientes com hipermetropia de }+3,25 \mathrm{a}+6,00 \mathrm{D}(\mathrm{Grupo} 2) \text {, submetidos à } \\
\text { cirurgia de LASIK, com o Sistema Ladar Vision de excimer laser. Acuidade } \\
\text { visual sem correção, melhor acuidade visual corrigida e refração sob } \\
\text { cicloplegia foram avaliadas em um, três e seis meses de pós-operatório. } \\
\text { Resultados: No grupo } 1, \text { o equivalente esférico médio pré-operatório, sob } \\
\text { cicloplegia, era de }+2,14 \pm 0,64 \mathrm{D} \text {, passando para }+0,44 \pm 0,38 \mathrm{D} \text { no sexto } \\
\text { mês de pós-operatório. No grupo } 2, \text { o equivalente esférico médio pré-ope- } \\
\text { ratório era de }+4,26 \pm 0,75 \mathrm{D} \text {, diminuindo para }+1,14 \pm 0,63 \text { D no sexto mês } \\
\text { de pós-operatório. } 3,4 \% \text { dos olhos do grupo } 2 \text { perderam três linhas de visão } \\
\text { no primeiro mês de pós-operatório. No grupo } 1, \text { não houve perda de duas } \\
\text { ou mais linhas de visão. Conclusões: O LASIK hipermetrópico com o } \\
\text { sistema Ladar Vision mostrou-se procedimento eficaz e seguro. Pacientes } \\
\text { do grupo } 2 \text { parecem estar sob maior risco de perda de linhas de melhor } \\
\text { acuidade visual corrigida no pós-operatório. }\end{array}$ \\
\hline
\end{tabular}

Descritores: Ceratomileuse assistida por excimer laser in situ; Hiperopia/cirurgia; Erros de refração/cirurgia; Cirurgia a laser/métodos; Resultado de tratamento

\section{INTRODUÇ̃̃̃O}

A correção fotorrefrativa da hipermetropia por meio da técnica de LASIK tem-se mostrado um desafio quando comparada ao tratamento miópico ${ }^{(1)}$, no que se refere à eficácia, previsibilidade e estabilidade do procedimento $^{(2)}$. Embora apresente várias vantagens em relação a outras técnicas, o LASIK hipermetrópico (H-LASIK) ainda cursava, até bem pouco tempo, com uma alta taxa de reoperações ${ }^{(3)}$. A literatura mostra que regressão significativa do tratamento pode ocorrer, mesmo em hipermetropias leves e modera$\operatorname{das}^{(4)}$.

Isto ocorre, em parte, porque, sendo a ablação hipermetrópica realizada na meia-periferia corneana, com o objetivo de aplanar a periferia e provocar secundariamente um encurvamento da região central, zonas de ablação pequenas utilizadas anteriormente, bem como lamelas pequenas produzidas por microcerátomos mais antigos, contribuíam para diminuição ou mesmo para a perda do efeito óptico desejado ${ }^{(3)}$. 
60 Resultados a curto prazo de ceratotomia lamelar pediculada (LASIK) para correção de hipermetropia com o sistema Ladar Vision de excimer laser

Além disso, o uso de zonas ópticas pequenas, utilizadas com sucesso para tratamentos miópicos, torna ainda mais crítica a ocorrência de descentralizações, podendo levar a aberrações e instabilidade refracional, principalmente em condições mesópicas, quando ocorre dilatação pupilar ${ }^{(2)}$. Outro fator importante para o sucesso dos procedimentos fotorrefrativos hipermetrópicos, além de uma zona de ablação maior, é a presença de zona periférica de transição de pelo menos $1,5 \mathrm{~mm}$, para prevenir regressão e melhorar a qualidade e a estabilidade do tratamento ${ }^{(4)}$.

O aparelho "Ladar Vision" produzido pela Summit Autonomous (Alcon Surgical, Orlando, FL, EUA) integra a última geração de excimer laser, tendo sido aprovado pelo FDA para o tratamento de hipermetropia até $+6,00 \mathrm{D}^{(5)}$. Estudos realizados sugerem vantagens do uso deste aparelho, em relação a outros sistemas de excimer laser ${ }^{(6)}$, por apresentar sistema de liberação em varredura, puntiforme, realizando ablação asférica, com zona de transição mais suave, maior diâmetro de zona de ablação, além de dispor de mecanismos de controle da centralização ("eye tracking") (7). Tais características parecem melhorar o perfil da ablação hipermetrópica, podendo diminuir a incidência de perda de linhas de visão causada por descentralização do tratamento e de sintomas como "glare" e halos relacionados ao perfil de ablação paracentral e à zona de transição.

O presente trabalho tem por objetivo avaliar retrospectivamente a eficácia e a segurança do LASIK hipermetrópico utilizando-se o sistema "Ladar Vision".

\section{MÉTODOS}

Foi realizado estudo retrospectivo, no qual 57 olhos de 35 pacientes portadores de hipermetropia primária foram submetidos à cirurgia fotorrefrativa por meio da técnica de LASIK, entre julho de 2001 e maio de 2002, na unidade de Cirurgia Refrativa do Departamento de Oftalmologia da Universidade Federal de São Paulo - UNIFESP- EPM. O aparelho utilizado para a realização das cirurgias foi o "Ladar Vision", produzido pela "Summit Autonomous". Os pacientes incluídos no estudo apresentavam refração, sob cicloplegia, com componente esférico de $+1,00$ a $+6,00 \mathrm{D}$ e cilindro até - $0,75 \mathrm{D}$; foram subdivididos em dois grupos de acordo com a magnitude da hipermetropia tratada. No grupo 1, foram incluídos 28 olhos de 17 pacientes com equivalente esférico (EE) pré-operatório de $+1,00 \mathrm{D} \mathrm{a}+3,00 \mathrm{D}$ (hipermetropia baixa), e, no grupo 2, 29 olhos de 18 pacientes com EE pré-operatório de $+3,25$ a $+6,00 \mathrm{D}$ (hipermetropia moderada). No grupo 1, dos 28 olhos, 24 $(85,7 \%)$ foram acompanhados por um mês, $23(82,1 \%)$ por três meses e $22(78,6 \%)$ por seis meses de pós-operatório. No grupo 2, dos 29 olhos, $100 \%$ foram acompanhados por um mês, $11(37,9 \%)$ por três meses, e $15(51,7 \%)$ por seis meses de pósoperatório.

A avaliação pré-operatória consistiu de medidas da acuidade visual com e sem correção pela tabela de Snellen, refra- ção estática sob cicloplegia com ciclopentolato a $1 \%$, refração dinâmica, medida do diâmetro pupilar em ambiente iluminado e em condições mesópicas, biomicroscopia, tonometria de aplanação e oftalmoscopia indireta. Foram realizados ainda topografia corneana computadorizada (Eye Sys, Houston, TX, EUA), paquimetria ultra-sônica (Humphrey, San Leandro, CA, EUA) e/ou paquimetria óptica por fenda (Orbscan II, Salt Lake City, EUA).

Os pacientes usuários de lentes de contato foram orientados a suspender seu uso por, no mínimo, três semanas antes do exame inicial, no caso de lentes rígidas, e por uma semana, quando se tratava de lentes gelatinosas. Tal avaliação inicial só foi realizada nos casos em que sinais topográficos de irregularidade corneana, devido ao uso de lentes de contato, foram descartados. As cirurgias foram realizadas por médicos estagiários (no total de 11) do Setor de Cirurgia Refrativa da Universidade Federal de São Paulo-UNIFESP-EPM, tendo sido devidamente acompanhadas pelos médicos orientadores do Setor. Todas as cirurgias foram programadas baseando-se na refração total dos pacientes sob cicloplegia, utilizando-se a correção por meio de nomograma proposto pelo FDA, com base em análises iniciais de olhos tratados em estudos anteriores. Todas as cirurgias foram realizadas visando à emetropia.

Os pacientes portadores de patologias oculares, cirurgias oculares prévias, diabetes mellitus ou doenças auto-imunes, bem como pacientes com espessura corneana central, medida por paquimetria ultra-sônica e/ou orbscan II, com valor que não permitisse manutenção de leito estromal residual mínimo de $250 \mu$ após tratamento, com lamela de $160 \mu$, tiveram suas cirurgias contra-indicadas. De acordo com a rotina estabelecida no Serviço, pacientes com menos de 35 anos e/ou pacientes que apresentavam diferença entre a refração sob cicloplegia e refração dinâmica $>1,00$ D não foram submetidos à cirurgia e não fazem parte deste estudo. Para indicação da cirurgia verificaram-se a estabilidade refracional de pelo menos um ano, e a melhor AV corrigida pré-operatória maior ou igual a 20/40. Os pacientes receberam explicações sobre os procedimentos, riscos, e eventuais complicações da cirurgia de LASIK hipermetrópico, e todos assinaram o termo de consentimento informado padrão, utilizado no Serviço.

Durante a preparação para a cirurgia, os pacientes foram submetidos a um registro fotográfico que estabelece o centro da pupila não dilatada em relação ao limbo, onde é centralizada a zona de ablação, recurso presente no sistema de excimer laser do Ladar Vision. O registro fotográfico feito pelo aparelho tem por finalidade identificar a área a ser tratada, centralizando-a em relação ao eixo visual do paciente. Tanto a fotografia quanto à cirurgia foram realizadas estando o olho contra lateral coberto. Foi realizada então dilatação pupilar, de pelo menos $7 \mathrm{~mm}$, com uma mistura de fenilefrina a $2,5 \%$ e tropicamida a $1,0 \%$, necessária para o acionamento do " eye tracker", dispositivo presente no Ladar Vision que permite ao laser acompanhar eventuais movimentos oculares ocorridos durante a realização da fotoablação. A taxa de repetição do laser é de $61 \mathrm{~Hz}$, a fluência média, de 180 a $240 \mathrm{~mJ} / \mathrm{cm}^{2} \mathrm{e}$ a 
energia varia de 2,4 a 3,0 mJ. Nos casos em que houve correção associada de astigmatismo hipermetrópico (até $0,75 \mathrm{D}$ ), foram realizadas, previamente, à lâmpada de fenda, marcas com violeta de genciana, às 3 e às 9 horas, na região límbica. Tais marcas são utilizadas pelo programa do aparelho como uma referência do eixo de $180^{\circ}$, para corrigir eventuais torções oculares ocasionadas pela posição supina, minimizando a possibilidade de erro na correção do eixo do astigmatismo.

Os microcerátomos utilizados foram hansatome com anel 9, 5, Moria M2 e Moria Carriazo Barraquer, tendo-se o cuidado de obter, de acordo com o nomograma de cada microcerátomo, a maior lamela possível para cada caso. Nos casos em que foi verificada superposição da zona de ablação e da região do pedículo, esta foi protegida utilizando-se máscara disponível no Ladar Vision. Zona óptica de 6,0 mm e transição de 1,5 mm, com zona total de ablação de $9,0 \mathrm{~mm}$ foram utilizadas em todos os casos, seguindo o padrão de ablação hipermetrópica do aparelho.

A medicação pós-operatória consistiu em colírios com combinação de tobramicina e dexametasona quatro vezes ao dia, por 14 dias, e lágrima artificial por três meses, quatro vezes ao dia. Os pacientes foram orientados a utilizar protetores oculares acrílicos durante as cinco primeiras noites no pósoperatório. Nenhum paciente foi submetido à cirurgia bilateral simultânea, como parte da rotina do Serviço. Como não houve casos de retratamento, todos os resultados referem-se a um único procedimento. Avaliações posteriores foram realizadas no primeiro dia, em uma semana, um, três, e seis meses de pósoperatório. Foram realizadas medidas de acuidade visual sem correção, avaliação biomicroscópica, refração sob cicloplegia e acuidade visual corrigida, em todas as visitas, a partir do primeiro mês de pós-operatório. Os resultados de um, três e seis meses foram analisados separadamente para cada grupo e comparados entre si, no que se refere à eficácia e à segurança do procedimento.

\section{RESULTADOS}

Dos 35 pacientes deste estudo, $23(65,7 \%)$ pertenciam ao sexo feminino, e $12(34,3 \%)$ ao sexo masculino. A média de idade dos pacientes foi de 49,5 anos, variando de 35 a 63 anos. No grupo 1, a média do equivalente esférico sob cicloplegia no pré-operatório foi de $+2,14 \pm 0,64$, variando de $+1,00 \mathrm{a}+3,00 \mathrm{D}$, e a média do EE sob cicloplegia em seis meses de pós-operatório foi de $+0,44 \pm 0,38 \mathrm{D}$, variando de $-0,50 \mathrm{a}+1,25$. No grupo 2 , a média do EE pré-operatório foi de $+4,26 \pm 0,75 \mathrm{D}$, variando de $+3,25 \mathrm{a}+6,00$, passando para $+1,14 \pm 0,63 \mathrm{D}$ no sexto mês pósoperatório, com variação de $+0,25 \mathrm{a}+2,50 \mathrm{D}$. (Tabela 1 ). Os resultados dos grupos 1 e 2 foram comparados entre si, instante a instante, por meio de um modelo de análise de variância, com medidas repetidas (ANOVA), que mostrou diferença estatisticamente significante entre os grupos no pré-operatório, e no sexto mês de pós-operatório, momentos em que a média do EE do grupo 2 foi estatisticamente maior que a do grupo 1.

\begin{tabular}{|c|c|c|c|c|c|}
\hline Grupo & & EE pré & EE 1m & EE 3m & EE 6m \\
\hline \multirow[t]{4}{*}{1} & Média & $+2,14^{*}$ & $+0,25$ & $+0,31$ & $+0,44^{* *}$ \\
\hline & Desvio padrão & 0,64 & 0,43 & 0,49 & 0,38 \\
\hline & Mínimo & $+1,00$ & $-0,75$ & $-0,75$ & $-0,50$ \\
\hline & Máximo & $+3,00$ & $+1,00$ & $+1,50$ & $+1,25$ \\
\hline \multirow[t]{4}{*}{2} & Média & $+4,26^{*}$ & $+0,95$ & $+0,79$ & $+1,14^{* *}$ \\
\hline & Desvio padrão & 0,75 & 0,69 & 0,56 & 0,63 \\
\hline & Mínimo & $+3,20$ & $-0,50$ & 0,00 & $+0,25$ \\
\hline & Máximo & $+6,00$ & $+2,00$ & $+2,00$ & $+2,50$ \\
\hline \multirow[t]{4}{*}{ Total } & Média & $+3,23$ & $+0,63$ & $+0,47$ & $+0,72$ \\
\hline & Desvio padrão & 1,28 & 0,68 & 0,56 & 0,60 \\
\hline & Mínimo & $+1,00$ & $-0,75$ & $-0,75$ & $-0,50$ \\
\hline & Máximo & $+6,00$ & $+2,00$ & $+2,00$ & $+2,50$ \\
\hline \multicolumn{6}{|c|}{ EE: equivalente esférico; ${ }^{*}: p<0,001 ;{ }^{* *}: p<0,001$} \\
\hline
\end{tabular}

Nas avaliações de um e três meses, do ponto de vista estatístico, os grupos não se mostraram distintos.

Ainda como parte da avaliação da eficácia do procedimento, comparamos os valores da correção esperada com os valores da correção obtida para cada grupo em um mês, e em seis meses de pós-operatório. Para análise da concordância dessas duas medidas de natureza numérica foram construídos gráficos de Bland-Altman (Gráficos 1, 2, 3 e 4) que mostram a diferença entre a correção esperada e a correção obtida (EE residual), segundo a média do EE pré-operatório, em um e seis meses de pós-operatório, para cada grupo. Foram calculados coeficientes de correlação intraclasse. (Tabela 2).

No grupo 1, dos olhos avaliados no primeiro mês de pósoperatório, $75 \%$ estavam com EE entre $\pm 0,50 \mathrm{D}$ e $87,5 \%$ entre $\pm 1,00$ D. No grupo 2, neste mesmo período, $44,8 \%$ dos olhos estavam com EE entre $\pm 0,50 \mathrm{D}$ e $65,5 \%$ entre $\pm 1,00 \mathrm{D}$. Ainda no primeiro mês de pós-operatório, $62,5 \%$ dos olhos no grupo 1 apresentavam AV sem correção (AV s/c) igual, ou melhor, que 20/20, e 100\% apresentavam (AV s/c) igual, ou melhor, que

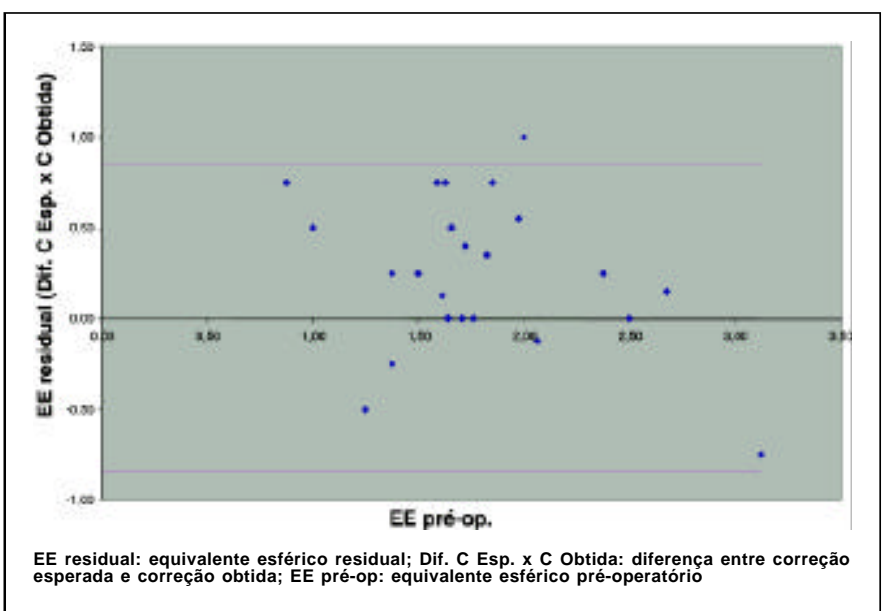

Gráfico 1. Comparação entre a correção esperada e a correção obtida (Gráfico de Bland-Altman), para o grupo 1 no mês 1 ( $n=24)$ 


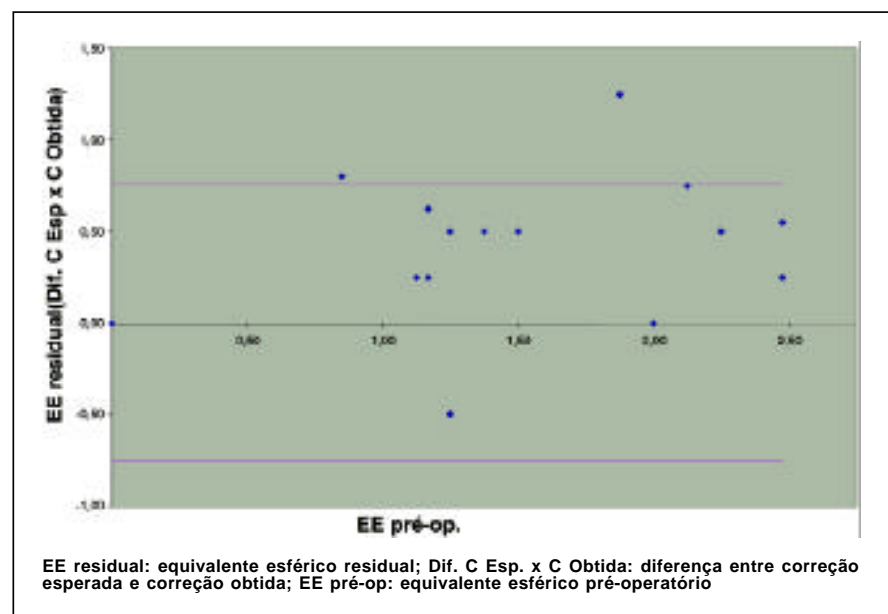

Gráfico 2. Comparação entre a correção esperada e a correção obtida (Gráfico de Bland-Altman), para o grupo 1 no mês 6 ( $n=22)$

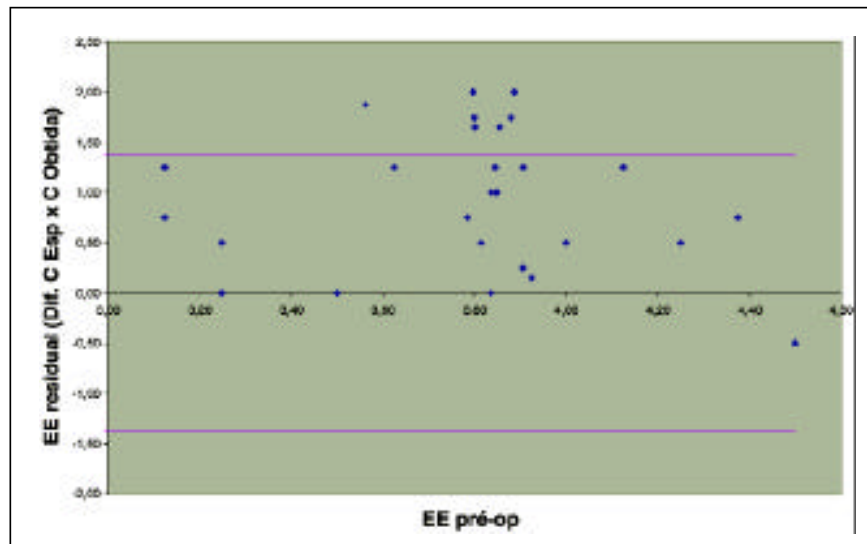

EE residual: equivalente esférico residual; Dif. C Esp. x C Obtida: diferença entre correção esperada e correção obtida; EE pré-op: equivalente esférico pré-operatório

Gráfico 3. Comparação entre a correção esperada e a correção obtida (Gráfico de Bland-Altman), para o grupo 2 no mês $1(n=29)$

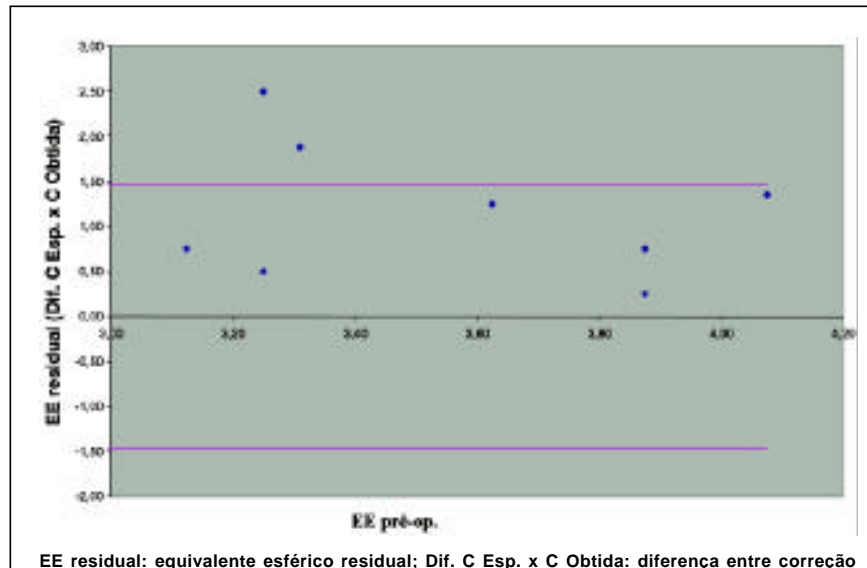
EE residual: equivalente esférico residual; Dif. C Esp. $x$ C Obtida: diferença entre correção

Gráfico 4 . Comparação entre a Correção Esperada e a Correção Obtida (Gráfico de Bland-Altman), para o Grupo 2 no mês $6(n=15)$

\begin{tabular}{|lcccc|}
\hline \multicolumn{4}{|c|}{$\begin{array}{c}\text { Tabela 2. Coeficientes de correlação intraclasse calculados para } \\
\text { um e seis meses de pós-operatório, segundo grupo }\end{array}$} \\
Grupo & Mês & Coeficiente & IC (95\%) \\
1 & 1 & 0,7633 & 0,$5310 ; 0,8890$ \\
1 & 6 & 0,6210 & 0,$2863 ; 0,8220$ \\
2 & 1 & 0,0361 & $-0,3251 ; 0,3901$ \\
2 & 6 & $-0,3225$ & $-0,6990 ; 0,2035$ \\
\hline IC: intervalo de confiança & & & \\
\hline
\end{tabular}

20/40. No grupo 2, 34,5\% apresentavam (AV s/c) igual, ou melhor, que 20/20, e 86,2\% apresentavam (AV s/c) igual, ou melhor, que 20/40.

$\mathrm{Na}$ avaliação pós-operatória de seis meses, $50 \%$ dos olhos do grupo 1 apresentavam EE entre $\pm 0,50 \mathrm{D}, 77,3 \%$ entre $\pm 1,00$ D, 40,9\% apresentavam (AV s/c) melhor ou igual a 20/20, e $95,5 \%$ melhor ou igual a 20/40. No grupo 2, dos olhos avaliados após seis meses de cirurgia, 26,7\% apresentavam EE entre $\pm 0,50 \mathrm{D}, 53,3 \%$ entre $\pm 1,00 \mathrm{D}, 46,7 \%$ apresentavam $\mathrm{AV}$ s/c melhor ou igual a 20/20, e 93,3\% melhor ou igual a 20/40.

Para análise da estabilidade do equivalente esférico pósoperatório ao longo do tempo, foram utilizados apenas os olhos avaliados em todos os retornos (em um, três e seis meses); os resultados de cada grupo foram comparados instante a instante, por meio de um modelo de análise de variância com medidas repetidas (ANOVA). Embora se tenha evidenciado discreto aumento no EE pós-operatório ao longo do tempo, especialmente no grupo 2, essa diferença não se mostrou estatisticamente significante em nenhum dos grupos (Gráfico 5).

Nenhum dos pacientes avaliados neste estudo foi submetido a reoperação.

A segurança do procedimento foi avaliada no que se refere à perda de linhas de melhor acuidade visual corrigida no pósoperatório. No grupo 1, nenhum dos olhos avaliados apresentou perda de duas ou mais linhas em qualquer período pósoperatório, diferindo do grupo 2 , no qual um dos olhos $(3,4 \%)$ apresentou perda de três linhas de visão no primeiro mês de

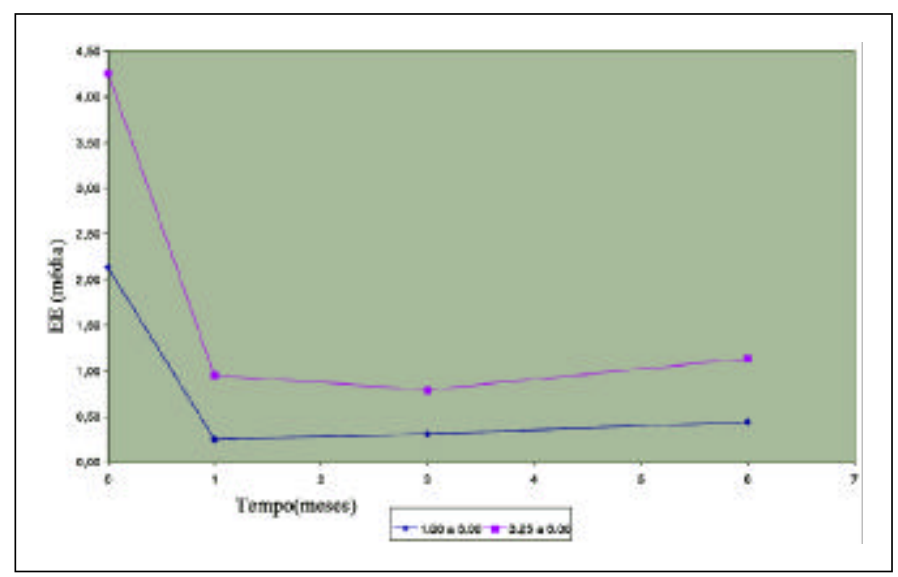

Gráfico 5. Evolução da média dos equivalentes esféricos ao longo do tempo, segundo grupo 
pós-operatório. No grupo 1, 8,3\% dos olhos tiveram perda de uma linha de visão no primeiro mês e $18,2 \%$ no sexto mês de pós-operatório. Já no grupo 2, 24,1\% dos olhos perderam uma linha de visão no primeiro mês, e 26,7\% no sexto mês. Embora se evidencie maior proporção de perda de linhas no grupo 2 , não houve diferença estatisticamente significante entre os grupos, pelo teste de comparação de duas proporções $(p=0,127)$. Não houve casos de descentralização do tratamento comprovados por topografia diferencial, nem complicações que exigissem reintervenção, como epitélio na interface, dobras significativas na lamela ou infecção.

\section{DISCUSSÃO}

A magnitude da hipermetropia pré-operatória, o poder ceratométrico prévio e o diâmetro da zona de ablação são fatores que sabidamente influenciam a eficácia e a estabilidade em longo prazo do lasik hipermetrópico. Hipocorreção e regressão ocorrem mais freqüentemente quando são utilizadas zonas de ablação menores que $6,0 \mathrm{~mm}$ e em tratamentos de hipermetropias altas ou moderadas ${ }^{(8)}$.

Zadok e colaboradores, analisando 72 olhos submetidos a H-Lasik, observaram boa previsibilidade no tratamento de hipermetropia até $+3,00 \mathrm{D}$, com $89 \%$ dos olhos entre $\pm 1,00 \mathrm{D}$ e diminuição da previsibilidade no grupo de $+3,00$ a $+5,00 \mathrm{D}$ $(52 \% \text { entre } \pm 1,00 \mathrm{D})^{(4)}$.

Estes dados estão de acordo com o observado no presente trabalho, em que $87,5 \%$ dos olhos no grupo 1 , e $65,5 \%$ dos olhos no grupo 2, apresentavam EE entre $\pm 1,00 \mathrm{D}$ no primeiro mês de pós-operatório. Embora se verifique uma tendência à diminuição da eficácia e previsibilidade do procedimento no grupo de hipermetropia moderada, quando comparada ao grupo de hipermetropia baixa, esta diferença entre os grupos não foi estatisticamente significante, no teste para comparação de duas proporções, provavelmente pelo tamanho reduzido da amostra.

Argento e Cosentino mostraram que os resultados de HLasik com zona óptica de 5,9 mm são mais estáveis, previsíveis e seguros que aqueles realizados com zonas ópticas menores que $5,9 \mathrm{~mm}^{(9)}$. Para atender a esta necessidade, já está disponível no mercado, e com uso bastante difundido, uma nova geração de microcerátomos capazes de criar lamelas de diâmetros maiores $^{(7)}$, para que o leito estromal possa abrigar uma zona óptica de $6,0 \mathrm{~mm}$, e zona de transição de $1,5 \mathrm{~mm}$, totalizando uma zona de ablação de 9,0 mm. Em lamelas menores, se parte da ablação é realizada fora do leito estromal, pode ocorrer posterior crescimento e hiperplasia epitelial na região não protegida pela lamela, levando à regressão do efeito obtido, de forma semelhante ao que se verifica na ceratectomia fotorrefrativa (PRK) para tratamento de hipermetropia ${ }^{(10)}$, motivo que contribuiu para o abandono desta técnica. Igualmente importante para evitar a regressão é o perfil da zona de transição, que deve ser suave para que a presença de degraus não estimule a hiperplasia epitelial sob a lamela.
Outro fator a ser considerado no planejamento de correções hipermetrópicas é a ceratometria pós-operatória esperada. Choi e Wilson limitam a indicação de H-lasik a hipermetropias de, no máximo $+5,00 \mathrm{D}$, e aos casos em que a curvatura corneana pós-operatória esperada seja de no máximo $49,0 \mathrm{D}$, por temer uma diminuição da qualidade de visão, e um risco aumentado de astigmatismo irregular, quando correções acima destes níveis são realizadas ${ }^{(11)}$. Como parte da rotina do nosso Serviço, e pelos mesmos motivos acima mencionados, contraindicamos qualquer procedimento corneano em hipermetropias acima de $+6,00 \mathrm{D}$, ou casos em que a ceratometria pósoperatória estimada seja superior a 49,0 D. Astigmatismo irregular pode resultar também de descentralização do tratamento, levando a sintomas subjetivos de diplopia monocular e má qualidade de visão(11).

O aparelho LADAR Vision, produzido pela Summit Autonomous, apresenta características especialmente importantes para a obtenção de um bom perfil de tratamento hipermetrópico: maior zona de ablação $(6,0 \mathrm{~mm})$, zona de transição suave $(1,5 \mathrm{~mm})$, sistema de rastreamento puntiforme, realizando ablação asférica, além de dispor de mecanismo de centralização ("eye tracking”), capaz de monitorizar, em uma freqüência de 4000 vezes por segundo, a posição ocular, compensando eventuais movimentos oculares involuntários ocorridos durante a ablação e minimizando, desta forma, o risco de descentralização do tratamento ${ }^{(7)}$.

Os resultados obtidos neste trabalho foram comparáveis aos de recente estudo multicêntrico prospectivo, realizado com o sistema Ladar Vision de excimer laser ${ }^{(7)}$.

Ao compararmos a correção obtida com a correção esperada, observamos tendência de hipocorreção da hipermetropia em ambos os grupos, tendo sido esta mais acentuada no grupo de hipermetropia moderada. É necessário salientar, entretanto, que nossos resultados se baseiam em refrações pós-operatórias sob cicloplegia. Provavelmente haveria maior concordância entre resultados esperados e obtidos se fosse realizada apenas refração manifesta no pós-operatório, (como se observa em muitos trabalhos na literatura $\left.{ }^{(2,4,11-12)}\right)$, visto que os pacientes, embora présbitas em sua maioria, apresentam alguma capacidade de acomodação, como mostram nossos bons resultados referentes à acuidade visual sem correção pós-operatória.

Os bons resultados obtidos, especialmente no grupo de hipermetropia baixa, devem ser atribuídos, em parte, à seleção dos pacientes, na qual a idade maior que 35 anos e a proximidade entre a refração estática e a dinâmica (diferença de 1,00 D no máximo) foram fatores decisivos para a indicação da cirurgia. A rotina adotada por nosso Serviço é a de tratamento total da refração obtida sob cicloplegia, o que poderia levar a maus resultados em pacientes jovens.

Quanto à estabilidade, embora possamos observar discreto aumento do EE médio ao longo do tempo, especialmente no grupo 2 (Gráfico 5), não houve diferença estatisticamente significante entre um e três, três e seis, e um e seis meses, em nenhum dos grupos. Entretanto, em virtude do curto tempo de 
seguimento (seis meses) não julgamos estes resultados suficientes para atestar a estabilidade do procedimento. Estudo prospectivo com o sistema Ladar Vision de excimer laser, com tempo de seguimento de 1 ano demonstraram a estabilidade do lasik hipermetrópico ${ }^{(7)}$.

Quanto à segurança do procedimento, no que se refere à perda de linhas da melhor AV corrigida pós-operatória, em relação ao pré-operatório, não observamos perda de duas ou mais linhas no grupo 1, enquanto perda de três linhas foi verificada em $3,4 \%$ dos olhos no grupo 2 . A perda de uma linha de visão ocorreu em ambos os grupos, em maior proporção no grupo 2, tanto no primeiro como no sexto mês de pós-operatório, o que também se observa em trabalhos publicados na literatura, que apontam aumento da magnitude da hipermetropia a ser corrigida como fator de risco para perda de linhas da melhor AV corrigida pós-operatória ${ }^{(2,11)}$. Não se observou diferença estatisticamente significante entre os grupos no que se refere à perda de linhas de visão, pelo teste de comparação de duas proporções, provavelmente pelo tamanho reduzido da amostra.

Além dos resultados avaliados objetivamente, dados relativos a queixas subjetivas referidas pelos pacientes, não incluídos neste trabalho, mostram que apenas $5,8 \%$ dos pacientes do grupo 1 e $11,1 \%$ do grupo 2 apresentaram queixas leves (grau 1 em escala de 0 a 4 ) de baixa acuidade visual para longe, "glare" e perturbações visuais noturnas, nenhum deles tendo solicitado reoperação.

Por considerar que os pacientes hipermétropes présbitas, em sua maioria, não são usuários de lentes de contato, que alguns não se adaptam bem aos óculos bifocais ou multifocais, e que a cirurgia, além de melhorar sensivelmente a $\mathrm{AV}$ s/c para longe, diminui a refração total que estes pacientes necessitam para perto, torna-se fácil entender por que temos observado pacientes satisfeitos, independentemente de uma $\mathrm{AV} \mathrm{s} / \mathrm{c}$ pós-operatória de 20/20, 20/30, ou 20/40.

\section{CONCLUSÃO}

A análise dos resultados obtidos, bem como os avanços tecnológicos desenvolvidos, visando a melhoria dos resultados do LASIK hipermetrópico, levam-nos a crer que, respeitando-se certos critérios, a cirurgia refrativa para hipermetropia com o sistema Ladar Vision de excimer laser mostrou-se um procedimento eficaz e seguro para correção de hipermetropias esféricas de $+1,00$ a +6,00 D. O grupo de hipermetropia moderada, quando comparado ao de hipermetropia baixa, parece estar sob maior risco de perda de duas ou mais linhas de visão corrigida no pós-operatório, tendendo a apresentar resultados menos eficazes e previsíveis.

\section{ABSTRACT}

Purpose: To analyze the efficacy and safety of hyperopic laser in situ keratomileusis using the Ladar Vision excimer laser system. Methods: Twenty-eight eyes of 17 patients with hyperopia from +1.00 to $+3.00 \mathrm{D}$ (Group 1 ), and 29 eyes of 18 patients with hyperopia from +3.25 to $+6.00 \mathrm{D}$ (Group 2) that had LASIK for hyperopia with the Ladar Vision, were retrospectively analyzed. Uncorrected visual acuity, best spectaclecorrected visual acuity and cycloplegic refraction were evaluated 1, 3 and 6 months after surgery. Results: In group 1, the mean preoperative cycloplegic spherical equivalent (SE) was $+2.14 \pm 0.64 \mathrm{D}$ and 6-month postoperative $\mathrm{SE}$ was $+0.44 \pm 0.38$ D. In group 2 , the mean preoperative SE was $+4.26 \pm 0.75 \mathrm{D}$ and the 6-month postoperative SE was $+1.14 \pm 0.63$ D. $3.4 \%$ of the eyes in group 2 and none of the eyes in group 1 lost 2 or more lines of best spectacle-corrected visual acuity in the first postoperative month. Conclusions: LASIK with the Ladar Vision excimer laser system is an effective and safe procedure to correct hyperopia. Patients in group 2 appear to be at greater risk for loss of lines of best spectacle-corrected visual acuity.

Keywords: Keratomileusis, laser in situ; Hyperopia/surgery; Refractive errors/surgery; Laser surgery/methods; Treatment outcome

\section{REFERÊNCIAS}

1. Suarez E, Torres F, Duplessie M. LASIK for correction of hyperopia and hyperopia with astigmatism. Int Ophthalmol Clin 1996; 36:65-72.

2. Arbelaez MC, Knorz MC. Laser in situ keratomileusis for hyperopia and hyperopic astigmatism. J Refract Surg 1999, 15:406-14.

3. Ojeimi G, Waked N. Laser in situ keratomileusis for hyperopia. J Refract Surg 1997; 13 (5 suppl): S432-3.

4. Zadok D, Maskaleris G, Montes M, Shah S, Garcia V, Chayet A. Hyperopic laser in situ keratomileusis with the Nidek EC-5000 excimer laser. Ophthalmology 2000; 107:1132-7.

5. McDonald MB. Summit-Autonomous Custom Cornea laser in situ keratomileusis outcomes. J Refract Surg 2000; 16:S617-8.

6. Krueger RR. Technology requirements for Summit-Autonomous Custom Cornea. J Refract Surg 2000; 16:S592-601.

7. Salz JJ, Stevens C A. LASIK correction of spherical hyperopia, hyperopic astigmatism, and mixed astigmatism with the LADARVision excimer laser system. Ophthalmology 2002; 109:1647-57.

8. Esquenazi S, Mendoza A. Two-year follow-up of laser in situ keratomileusis for hyperopia. J Refract Surg 1999; 15:648-52.

9. Argento CJ, Cosentino MJ. Comparison of optical zones in hyperopic laser in situ keratomileusis: $5.9 \mathrm{~mm}$ versus smaller optical zones. J Cataract Refract Surg 2000; 26:1137-46.

10. McGhee CN, Ormonde S, Kohnen T, Lawless M, Brahma A, Comaish I. The surgical correction of moderate hypermetropia: the management controversy. Br J Ophthalmol 2002; 86:815-22.

11. Choi RY, Wilson SE. Hyperopic laser in situ keratomileusis: primary and secondary treatments are safe and effective. Cornea 2001; 20:388-93.

12. Davidorf JM, Eghbali F, Onclinx T, Maloney RK. Effect of varying the optical zone diameter on the results of hyperopic laser in situ keratomileusis. Ophthalmology 2001; 108:1261-5. 